\title{
Spontaneous parity breaking and fermion masses
}

\section{J. A. Martins Simões*†, Y. A. Coutinho and C. M. Porto}

Instituto de Física, Universidade Federal do Rio de Janeiro, RJ, Brazil and Universidade Federal Rural do Rio de Janeiro, RJ, Brazil

Abstract: We present a $S U(2)_{L} \otimes S U(2)_{R} \otimes U(1)$ left-right symmetric model for elementary particles and their connection with the fermion mass spectrum. New mirror fermions and a minimal set of Higgs particles are proposed. The model can accommodate a consistent pattern for charged and neutral fermion masses as well as neutrino oscillations. The connection between the left and right sectors can be done by the neutral vector gauge boson $Z$ and a new heavy $Z^{\prime}$.

One possible way to understand the origin of the left-right asymmetry in weak interactions is to enlarge the standard model into a left-right symmetric structure and then, by some spontaneously broken mechanism, to recover the low energy asymmetric world. Leftright models starting from the gauge group $S U(2)_{L} \otimes S U(2)_{R} \otimes U(1)_{B-L}$ were developed by many authors $[1]$ and are well known to be consistent with the standard $S U(2)_{L} \otimes U(1)_{Y}$. However, for the fermion mass spectrum there is no unique choice of the Higgs sector that can reproduce the observed values for both charged and neutral fermions, neither the fundamental fermionic representation is uniquely defined.

As the parity asymmetry is clearly displayed in the electroweak sector of the standard model, we take [2] as a minimal gauge sector the left-right symmetric group $S U(2)_{L} \otimes$ $S U(2)_{R} \otimes U(1)_{B-L}$, with generators $\left(T, T^{*}, Y\right)$. This group can be considered as a subgroup of many unification groups like the superstring inspired $E_{8} \otimes E_{8}^{\prime}$ or the SUSY $S O(10) \otimes S O(10)^{\prime}$. We will assume as a starting point, new mirror fermions with the following assignment:

$$
\ell_{L}=\left(\begin{array}{c}
\nu \\
e
\end{array}\right)_{L}, \nu_{R}, e_{R} \stackrel{\mathrm{P}}{\longrightarrow} L_{R}=\left(\begin{array}{c}
N \\
E
\end{array}\right)_{R}, N_{L}, E_{L}
$$

The other lepton and quark families follow a similar pattern. This fundamental representation clearly is anomaly free. In this model the parity operation transforms the $S U(2)_{L} \stackrel{\mathrm{P}}{\longleftrightarrow} S U(2)_{R}$ sectors, including the vector gauge bosons. This symmetry property

${ }^{*}$ Speaker.

${ }^{\dagger}$ simoes@if.ufrj.br 
can be used to reduce the three group constants $g_{L} ; g_{R} ; g^{\prime}$ to only two, with $g_{L}=g_{R}$. For the other leptonic and quark families a similar structure is proposed. The charge generator is given by $Q=T_{3}+T_{3}^{*}+Y / 2$.

In order to break $S U(2)_{L} \otimes S U(2)_{R} \otimes U(1)_{Y}$ down to $U(1)_{e m}$ we introduce two Higgs doublets that under parity are transformed as $\chi_{L} \leftrightarrow \chi_{R}$. Their quantum numbers are $(1 / 2,0,1)$ and $(0,1 / 2,1)$ respectively, with the corresponding vacuum parameters $v_{L}$ and $v_{R}$. The model also includes a Higgs field $\Phi$ in the mixed representation $(1 / 2,1 / 2,0)$ and new neutral singlets $S$ in $(0,0,0)$, broken at $s_{G U T}$ or at lower scales. The vacuum parameters are $k$ and $k^{\prime}$ for the $\Phi$ and $s$ for $S$.

In the standard model, Higgs doublets are responsible for the gauge boson masses as well as for the fermion masses. For the fermion masses the standard model requires adjusting by hand the Yukawa couplings in order to reproduce the observed mass spectrum. Although this procedure is consistent in the sense that all couplings satisfy $g_{i}<1$, there is no experimental confirmation for this hypothesis. One of the main points of our work is to show that Higgs singlets in left-right models can give a more natural charged and neutral fermion mass spectrum.

The basic physical assumptions of our model are the following. The consistency with the lower energy standard model sector $S U_{L}(2) \otimes U(1)$ is achieved if we suppose the symmetry breaking hierarchy $s_{G U T}>>v_{R}>>v_{L}>>k, k^{\prime}$. This was fully developed in ref. [2] and the main result is that we must have the bound $v_{R}>30 v_{L}$. The second assumption is that we must have some see-saw mechanism in the neutrino sector in order to have small neutrino masses. The third point is that the smallness of the electron ( and other charged lepton masses) must also be naturally explained and not adjusted by hand as it is the case for the standard model. As we will shown bellow this can be easily done in models with both left and right handed singlet fermionic states. This point is the fundamental reason for our choice of the particular fermionic representation given in equation (1).

The fermion mass spectrum depends both on the Higgs choice of the model and on the fundamental fermionic representation. A particular property of the model is the presence of left and right handed singlets in the fundamental representation. This means that we can add to the mass lagrangian new bare terms or new Higgs singlets which have no consequences on the vector gauge boson masses. We consider two new Higgs singlets, one that is coupled to Dirac terms in the mass lagrangian - $S_{D}$ - and the other that couples to Majorana terms - $S_{M}$. After spontaneous symmetry breaking they develop vacuum parameters $s_{D}$ and $s_{M}$, respectively.

The most general Yukawa lagrangian is given by,

$$
\begin{aligned}
\mathcal{L} & =f\left\{\overline{\ell_{L}} \chi_{L} \nu_{R}+\overline{L_{R}} \chi_{R} N_{L}\right\} \\
& +f^{\prime}\left\{\overline{\ell_{L}} \chi_{L} N_{L}^{C}+\overline{L_{R}} \chi_{R} \nu_{R}^{C}\right\} \\
& +g S_{M}\left\{\overline{N_{L}^{C}} N_{L}+\overline{\nu_{R}^{C}} \nu_{R}\right\} \\
& +g^{\prime} S_{D}\left\{\overline{\bar{\nu}_{R}} N_{L}\right\}+g^{\prime \prime} S_{D} \overline{e_{R}} E_{L} \\
& +f "\left\{\overline{\ell_{L}} \Phi L_{R}\right\}+\text { h.c. }
\end{aligned}
$$

For the neutral fermions, the lagrangian after symmetry breaking is 


$$
\begin{aligned}
\mathcal{L} & =f v_{L} \overline{\nu_{L}} \nu_{R}+f^{\prime} v_{L} \overline{\nu_{L}^{c}} N_{L}+f v_{R} \overline{N_{R}} N_{L} \\
& +f^{\prime} v_{R} \overline{N_{R}^{C}} \nu_{R}+g s_{M} \overline{N_{L}^{C}} N_{L} \\
& +g s_{M} \overline{\nu_{R}^{C}} \nu_{R}+g^{\prime} s_{D} \overline{\nu_{R}} N_{L}+f^{\prime \prime} k \overline{\nu_{L}} N_{R},
\end{aligned}
$$

For the charged fermions we have a similar lagrangian,

$$
\begin{aligned}
\mathcal{L} & =f v_{L} \overline{e_{L}} e_{R}+f v_{R} \overline{E_{R}} E_{L} \\
& +g " s_{D} \overline{e_{R}} E_{L}+f " k \overline{e_{L}} E_{R},
\end{aligned}
$$

The generalization for the other families is straightforward. The diagonalization is most easily done [3i] by introducing the self conjugated fields ( with $i, j=\nu, N) \chi_{i}=\psi_{i L}+\psi_{i L}^{C}$ and $\omega_{j}=\psi_{j R}+\psi_{j R}^{C}$.

We are considering in this paper that the order of magnitude of the fermionic mass spectrum is given by the symmetry breaking scales and their combinations. This is a departure from the standard model procedure of adjusting couplings to masses. So we are supposing that all couplings are of order one.

In the basis $\left(\chi_{\nu} ; \omega_{N} ; \chi_{N} ; \omega_{\nu}\right)$ the general neutrino mass matrix is:

$$
M_{\nu, N}=\left(\begin{array}{cccc}
0 & k / 2 & v_{L} & v_{L} / 2 \\
k / 2 & 0 & v_{R} / 2 & v_{R} \\
v_{L} & v_{R} / 2 & s_{M} & s_{D} / 2 \\
v_{L} / 2 & v_{R} & s_{D} / 2 & s_{M}
\end{array}\right)
$$

and the charged fermion mass matrix is:

$$
M_{e, E}=\left(\begin{array}{cccc}
0 & k^{\prime} & 0 & v_{L} \\
k^{\prime} & 0 & v_{R} & 0 \\
0 & v_{R} & 0 & s_{D} \\
v_{L} & 0 & s_{D} & 0
\end{array}\right)
$$

For this last case, we recover the Dirac formalism by the standard [풀] $\pi / 4$ rotations over the Majorana fields.

The recent SNO [in] and non-zero masses, but still leaving open some theoretical possibilities. In view of the present experimental situation we will not proceed to fit all the neutrino masses and mixings but we will look for solutions which could accommodate neutrinos with masses in the $10^{-2}-10^{-3} \mathrm{eV}$ range. We present two possible solutions, with $k=k^{\prime}=0$, which differ in the choice of the symmetry breaking parameters.

\section{- Model I}

In this model the Higgs singlets are both broken at $s_{G U T}$. For neutrinos we have the following masses

$$
\begin{aligned}
& m_{\nu 1}=v_{L}^{2} / s_{G U T} ; m_{\nu 2}=v_{R}^{2} / s_{G U T} \\
& m_{N 1} \simeq m_{N 2} \simeq s_{G U T}
\end{aligned}
$$


and for the charged fermions

$$
\begin{aligned}
m_{e} & =v_{L} v_{R} / s_{G U T} \\
m_{E} & =s_{G U T}
\end{aligned}
$$

Using $v_{L}=v_{\text {Fermi }}$ and $s_{G U T}=10^{16} \mathrm{GeV}$, we must have $v_{R}=10^{10}$ in order to obtain the correct value for the electron mass. The first generation mass spectrum is then given by $m_{\nu 1} \simeq 10^{-2} \mathrm{eV} ; m_{\nu 2} \simeq 10 \mathrm{TeV} ; m_{N 1} \simeq m_{N 2} \simeq 10^{16} \mathrm{GeV} ; m_{e} \simeq 1 \mathrm{MeV} ; M_{E} \simeq 10^{16}$ $\mathrm{GeV}$. The smallness of the electron mass is a consequence of a "see-saw" mass relation given by equation 6 . This is a departure for the standard model mechanism for fermion masses. There is no mixing between $\nu_{1}$ and $\nu_{2}$ and we have an example of a sterile neutrino coming from a new exotic sector. The presently observed neutrino mixing must come from a possible generation mixing in the general neutrino mass lagrangian. Standard charged quarks are also found to be in the $\mathrm{MeV}$ mass range, with $m_{q}=v_{L} v_{R} / s_{G U T}$. With this high value for $v_{R}$ we have no experimental accessible accelerator possibilities for new gauge vector bosons. For the Large Hadron Collider (LHC) it has recently been shown [i-jid that heavy neutrino production is limited to masses of a few hundreds of GeV. Heavy neutrinos with masses in the $\mathrm{TeV}$ region can be produced in the next generation of colliders.

The $v_{R}$ value consistent with the electron mass is of the order of the Peccei-Quinn

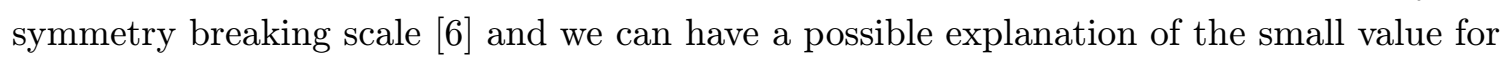
the $\theta$-angle of the strong $\mathrm{CP}$ problem, as shown in references [i]

In order to generate the other families mass spectrum we have the possibility of enlarging the Higgs singlet sector, postulating one new field for each family. In this last case we must have an hierarchy for neutrino masses.

\section{- Model II}

In this model lepton number is spontaneously broken at a scale $s_{M} \simeq s_{G U T}$ and the Higgs singlet coupled to Dirac mass terms is allowed to be broken at a lower scale. The fermion mass spectrum is given by

$$
\begin{aligned}
m_{\nu 1} & =v_{L}^{2} / s_{M} ; m_{\nu 2}=v_{R}^{2} / s_{M} \\
m_{N_{1}, N_{2}} & =s_{M} \pm s_{D} / 2 \\
m_{e} & =v_{L} v_{R} / s_{D} \\
m_{E} & =s_{D}
\end{aligned}
$$

If we take $v_{L}=v_{F e r m i}$ and $s_{M}=s_{G U T}$, then the electron and quark masses allow a solution given by $v_{R} \simeq 10^{4} \mathrm{GeV}$ and $s_{D} \simeq 10^{10} \mathrm{GeV}$. Here again we have a "see-saw" mechanism for the electron mass. In this model we could have an experimentally accessible new neutral current. One light neutrino has a mass in the $10^{-2}-10^{-3} \mathrm{eV}$ range and the other is in the $1-0.1 \mathrm{eV}$ region. These neutrinos are orthogonal and again we have a new sterile neutrino. The Peccei-Quinn symmetry breaking scale reappears in the Higgs singlet sector. Family replication can be recovered with different Dirac singlets and for only one Majorana mass scale we can have a degenerate neutrino mass spectrum.

The generalization for the other families can be easily extended from the above arguments. However, the mixing angle pattern is not so simple. A first approach is to general- 
ize the see-saw mechanism, with mixing angles given by the mass ratios $\theta_{\text {mix }} \simeq m_{\nu} / m_{N}$, which are very small numbers. There are many models that avoid such a restriction: the

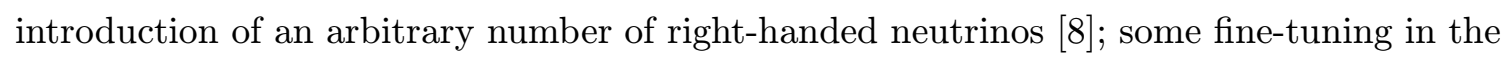
neutrino mass matrix $\left[\begin{array}{l}\overline{9} \\ \underline{9}\end{array}\right]$ and any general singular neutrino mass matrix can disconnect mixing parameters from mass ratios. So we will take mixing angles and neutrino masses as independent parameters.

In Model II we can have the production of new heavy neutral gauge bosons with mass scales accessible at new hadron colliders. Dilepton production in hadron-hadron collisions give a very clear signal for a new Z'. The Large Hadron Collider (LHC) facilities using proton-proton collisions at $\sqrt{s}=14 \mathrm{TeV}$, will attain higher $\mathrm{Z}$ ' masses in the $1-4 \mathrm{TeV}$ region. For an integrated luminosity at LHC of $100 \mathrm{fb}^{-1}$ and cuts of $E_{\ell}>20 \mathrm{GeV}$ and $|\eta|<2,5$ we expect 1000 events for $M_{Z^{\prime}}=1 \mathrm{TeV}$ and only one event for $M_{Z^{\prime}}=4 \mathrm{TeV}$.

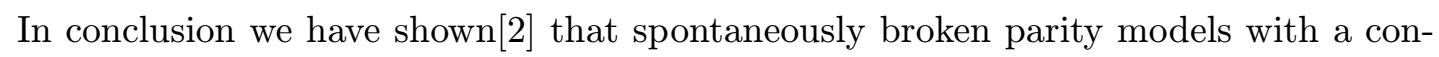
sistent fermion mass spectrum can be built at intermediate scales ranging from $10^{3}$ to $10^{10}$ $\mathrm{GeV}$. New mirror fermions are present and can be connected to ordinary fermions through neutral currents. We propose a simple Higgs sector for the model that allows several physically interesting solutions. The charged fermion masses can be generated by a "see-saw" mass relation, analogous to the neutrino sector. Various scenarios for neutrino masses are possible and a more clear experimental definition on neutrino masses will allow to test their reality. Neutral heavy gauge bosons Z and Z', coupled to ordinary and new mirror fermions, are expected to play a fundamental role in the understanding of the left-right symmetry. This is a departure from other models [i] $\left.\overline{0}_{1}\right]$ that propose gravitation as the connection between the left and right sectors. Experimental consequences of the model could be found at the next generation of colliders.

\section{Acknowledgments}

This work was supported by the Brazilian agencies: CNPq, FUJB, FAPERJ and FINEP.

\section{References}

[1] J.C.Pati and A.Salam, Phys. Rev. D 10 (1974) 275; R.N.Mohapatra and J.C.Pati,Phys. Rev. D 11 (1975) 566; G.Senjanovič and R.N.Mohapatra, Phys. Rev. D 12 (1975) 1502; R. N. Mohapatra and R.E. Marshak, Phys. Lett. B 91 (1980) 222; G. Senjanovič, Nuc. Phys. B 153 (1979) 334.

[2] Y.A. Coutinho, J.A. Martins Simões and C.M.Porto, Eur. Phys. J. C 18 (2001)779.

[3] T.P.Cheng and L.F.Li, Gauge Theory of Elementary Particle Physics. Oxford, 1984.

[4] Q.R. Ahmad et al, Nucl-Ex/ 0106015.

[5] Y.A.Coutinho, J.A.Martins Simões, P.P.Queiroz Filho, Phys. Rev D 57 (1998) 6975; F.M.L.Almeida Jr., Y.A.Coutinho, J.A.Martins Simões, M.A.B. Vale, Phys. Rev. D 62 (2000) 075004 (6 pages).

[6] J.E.Kim, Phys. Rep. 150 (1987) 1. 
[7] K.S.Babu and R.N.Mohapatra, Phys. Rev. D 41 (1990) 1286.

[8] C.Jarlskog, Phys. Lett. B 241 (1990) 579.

[9] W.Buchmüller and D.Wyler, Phys. Lett. B 249 (1990) 458; W.Buchmüller and C.Greub, Nuc. Phys. B 363 (1991) 345.

[10] I.Kobzarev, L.Okun and I.Ya Pomeranchuk, Sov. Jour. Nuc. Phys. 3 (1966) 837. 